\title{
ISSOR Signal Detection for Energy Efficient and Low Complexity Large Scale MIMO System
}

\author{
Seema M. Hanchate, Shikha Nema
}

\begin{abstract}
Scalable version of multiuser MIMO called Large-scale MIMO is a one of the powerful technology in future wireless communication systems in which huge amount of $B S$ (base station) antennas utilized to process multiple user equipment. Energy consumed is high with more antennas and also it leads to increase the signal detection complexity and overall circuit power consumption. Designing energy efficient and low complexity MIMO system is considered as a challenging issue. This paper presents the ISSOR signal detection for energy efficient and low complexity large scale MIMO system. VA-GSM (Variable Antenna Generalized spatial modulation) is used in which the number of active antenna transmissions are varied for every transmission in the large scale MIMO. In transmitter side, Eigen value based approach is used for antenna selection. Then, improved symmetric successive over relaxation (ISSOR) approach is proposed for low complexity signal detection in receiver side. The number of user equipment, transmit power, as well as the amount of antennas at the base station, are considered as the optimal system parameters which are chosen for enhancing the efficiency of utilized energy in the system. The proposed scheme implemented in MATLAB software. The proposed scheme attained the high energy efficiency compared to other approaches. Moreover, the BER is utilized to estimate the performance of an offered algorithm and also compared to the previously determined algorithm of existing literatures.
\end{abstract}

Keywords : Signal detection, antenna selection, energy efficiency, SOR, ISSOR

\section{INTRODUCTION}

T his is MIMO (Multiple input and multiple output) technology is able to enhance the capacity and reliability of wireless communication systems without use of additional bandwidth and transmit power. Therefore it is utilized in many future wireless standards as well as wireless systems [1]. At base station, huge amount of antennas $(>100)$ utilized in the large scale MIMO for attaining the finest enhancement in the efficiency of radiated energy as well as throughput. Most of the antennas present in the base station are set to focus the obtained energy on the spaces of ever- smaller regions. [2]. However, there are many different conditions have to be recognized to utilize the finest benefits of massive MIMO. Developing the algorithm for minimal-complexity

detection of energy efficient signal in uplink is the major challenging issue. [2]

Revised Manuscript Received on December 16, 2019.

* Correspondence Author

Seema M Hanchate*, Electronic \& Communication Department, Usha Mittal Institute of Technology, SNDT University, Mumbai, India. Email: smhancate.umit@gmail.com

Shikha Nema, Electronic \& Communication Department, Usha Mittal Institute of Technology, SNDT University, Mumbai, India.
The MIMO techniques will expand spectral efficiency and reliability through spatial multiplexing and spatial diversity respectively [3]. Spatial Modulation (SM) is known as the familiar modulation scheme which mostly utilized in the large scale MIMO. It reduces the necessity of multiple RF chains for transmission. To realize the spectral effectiveness, a single transmit antenna employed at every instance for transferring a guaranteed data symbol where the sending of data as well as the active antenna index are calculated according to the incoming data bits. [4]. Spatial modulation permits one active antenna for eliminating the ICI (Inter Channel Interference), nevertheless, decrease an applications of flexibility of design as well as spatial dimensions. GSM (Generalized Spatial modulation) is known as the evolved SM. This GSM permits different group of antennas to be active simultaneously. The GSM is used to exploit the spectral efficiency. It also enhanced energy efficiency by reducing number of RF chains [5].

According to the different data requirements, most of the joined devices are must be isolated for reconstructing the signals which are transmitted. The main investigation challenge is to proceed the signals which are obtained at the BS with less computational complexity [6]. Different signal detection algorithms are used to achieve the optimal signal detection and reduced complexity. ML (Maximum likelihood) is known as the optimal detector, increases the complexity with more antennas and hence becomes difficult to implement [7]. According to the SD (sphere decoder), consists only set of vectors to determine the ML solution with non-linear signal detection algorithm and difficult with high complexity. Another non-linear algorithm for the detection of signal used which related to TS (Tabu search) likewise the LTS (Layered Tabu search) algorithm [8].

Most of signal detection algorithms need complex matrix inversion. By new method with an expansion of Neumann series is used to reduce an issues of computational difficulty on large-scale MIMO systems in the uplink. The matrix inversion is converted by Neumann Series expansion into a sequence of matrix additions as well as multiplications. [9]. However the convergence speed is slow in Neumann Series[10].Based on Newton iteration (NI) the matrix inversion method, signal detection get low complexity. But the computation complexity is more for calculating proper initial input in Newton iteration [10].The existing works are faces the problem of high computational complexity and low energy efficiency during the transmission.

To reduce these kind of issues this work proposed an Improved Symmetric Successive Over relaxation (ISSOR) method for the signal detection. For antenna selection, this work implementing Eigen value based approach. This proposed algorithm increases 


\section{ISSOR Signal Detection for Energy Efficient and Low Complexity Large Scale MIMO System}

the energy efficiency of the large scale MIMO system.

The rest of this work is categorized as follows; section II describes about the existing work done by different researchers. The section III, brief about the system model of large scale MIMO and antenna selection algorithm. The section IV, explains the SOR and propose ISSOR method. In section $\mathrm{V}$, the energy efficiency of the proposed work is presented. In section VI, an outcomes of simulation of our proposed work is provided. Finally the section VII finalizes this work.

\section{RELATED WORK:}

A less critically iterative suboptimal detector method was proposed by Hugo lopes et al [11]. This algorithm process are unconstrained Euclidean distance minimization problem, over the signal constellation of projection in an element wise and projection over the bunch of accurate antenna combinations. The MIMO schemes are provided by GSM for accomplishing the finest energy efficiency as well as spectral efficiency. Furthermore, the scheme of MIMO able to be concerned as an agreement between Spatial multiplexing MIMO and conventional SM. The proposed algorithm gives better efficiency compared with existing works.

An iterative detection was proposed by Xiaochen He et al. [12] for the uplink. The channel matrix decompositions deliver the transformation matrices. For accurate iterative LMMSE detectors, transformed system model is developed using transformation matrices. The quadratic complexity required for the LMMSE (linear minimum mean squared error) detector in which more number of antennas also required. This proposed iterative LMMSE detectors, were achieved better performance as the conventional LMMSE detector. This work were also worthy to minimize the difficulty of downlink pre-coding. combining) signal detection algorithm proposed by Ben Ren et al. [13] according to the Eigen value decomposition of the noise variance as well as the interference. When conducting a comparison between the conventional algorithms with the suggested algorithm, the matrix inversion of computation-intensive of is reduced by the suggested algorithm. The computational complexity is focused in this work, this exhibited that the noise covariance matrix as well as inverse of the interference computed by the detection algorithm MMSE-IRC in uplink. While the quantity of antenna is maximized. This method provides the effective performance compared with other works.

For deliver the finest efficiency in energy as well as spectrum used in communication systems, Zhen Gao et al. [14] proposed a work. A low complexity signal detector is utilized in this work for downlink single-user massive SM-MIMO according to the SCS (Structured Compressive Sensing). In this proposed work, the massive low cost antennas are fitted for the massive SM- MIMO however at transmitter part, chains of less power-hungry transmit radio frequency (RF) are used. An algorithm of structured subspace pursuit (SSP) were presented in this work. At the part of the receiver, the structured sparsity is leveraged to detect as well as combine the multiple SM signals. This work attain better performance gain from the SM signal interleaving.

\section{SYSTEM MODEL OF LARGE SCALE VA-GSM} MIMO
A less critical MMSE-IRC (interference rejection

The large scale MIMO uses more number of service antennas that are operated adaptively and coherently. Additional antennas help to focus energy of the transmitted signal into ever-smaller regions of space. The VA GSM-MIMO selection module has the $\boldsymbol{P}_{T R}$ transmit antennas, $P_{L}$ active antennas, $P_{R E}$ receive antennas and also the constellation mapper exists of points $N$. The information bits are classified into groups in grouping module at the side of transmitting. Each group has $\mathrm{P}$ bits. Then the selected transmit antenna are given as input of the transmitter.

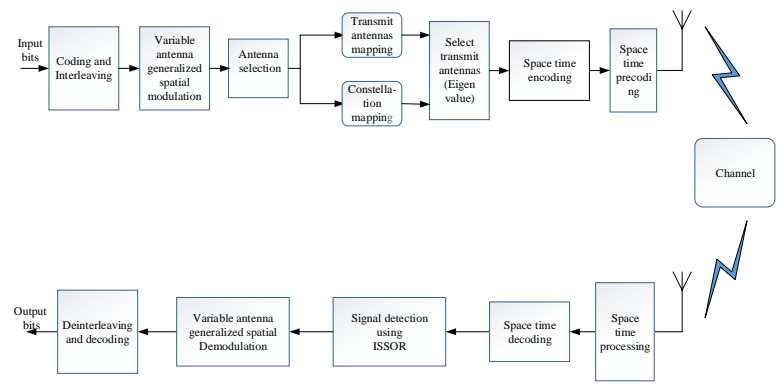

Fig 1. Block diagram of antenna selection and signal detection with VAGSM for large scale MIMO

The information bits are encoded by using a conventional encoder. The output of the encoding signal is to be interleaved. The interleaved symbols are modulated by using variable antenna generalized spatial modulation. Then the selection of antenna is performed by using Eigen value based approach. The output of antenna selection is an input to the space time encoding. It provide the one or more spatial data streams. The spatial data streams are mapped to the transmit antennas by space-time pre-coding block. The signals are transmit through the channel in selected antenna. The receiver collects the signals at the output of each receive antenna element and reverses the transmitter operations in order to decode the data through space-time processing and space-time decoding. The signal detection is done by ISSOR method without the complex matrix inversion. After, the variable antenna generalized spatial demodulation is used to demodulate the signal. Finally, the deinterleaving and decoding process is performed. The maximum number of bits which can be transmitted is denoted by $P$. The equation for $P$ is given below

$$
P=\left\lfloor\log _{2}\left(\begin{array}{c}
P_{T R} \\
P_{L}
\end{array}\right)\right\rfloor+\log _{2} N
$$

The floor function indicated as $\lfloor$.$\rfloor in above equation.$ The antenna combinations $2^{\left\lfloor\log _{2}\left(\begin{array}{c}P_{T R} \\ P_{L}\end{array}\right)\right\rfloor}$ chosen from the all

possibilities of $\left(\begin{array}{c}P_{T R} \\ P_{L}\end{array}\right)$ antenna combinations with suitable CSI estimated at receiver side in antenna selection module.

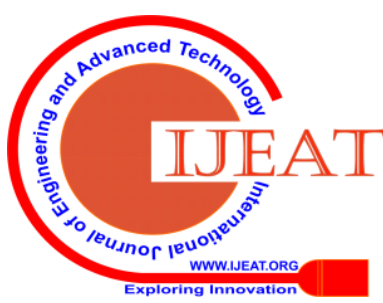


The point of the constellation, as well as the antenna combination, are decided according to the bit group, in which the similar constellation point is transmitted on the basis of $P_{L}$ chosen antennas. Over the gradual time varying channel $H$, the modulated signal is transmitted and there has no channel variations during the transmission period. Additionally, the Rayleigh flat fading channel dimension $H$ is $P_{R E} \times P_{T R}$. From $n-$ th transmission antenna to the $m$-th receiver antenna and also Gaussian random variable is denoted by every entry of $H$ and it is also identically distributed (i.i.d) as well as complex independent. After the signal passed via the channel, in which the following equation (2) taken for denote the received signal.

$$
y=\sqrt{\frac{E_{s}}{P_{L}}} \tilde{H}_{R} d+\lambda
$$

Where, $E_{s}$ denotes the average bit energy and $\tilde{H}_{R}=\left(H_{R}^{T} H_{R}\right)$ is the channel matrix $\left(P_{L} \times P_{L}\right)$ joined together with space and time domain. The set of potential subsets of transmit antennas are denoted by the notation $\mathrm{R}$ likewise $\boldsymbol{d}$ is the transmitted bit of information and $\mathrm{L}$ is resolvable paths as system parameters.

$$
\begin{gathered}
H_{R}=\left[H_{R}(0)^{T} H_{R}(1)^{T} \cdots H_{P}(L-1)^{T}\right]^{T} \\
\lambda=\sum_{l=0}^{L-1} H_{R}(l)^{T} \lambda(l) \\
\lambda(l)=\left[\lambda_{1}(l) \lambda_{2}(l) \cdots \lambda_{N_{R E}}(l)\right]^{T}
\end{gathered}
$$

Where $\lambda_{n}$ (l) denotes the white Gaussian noise vector with zero-mean at $\mathrm{n}^{\text {th }}$ receive antenna.

\section{A. Antenna Selection using Eigen value approach}

The natural characteristics of the Eigen space of the channel matrix is used in this algorithm. For clear view, assume the combined channel matrix for $P_{T R}=2, L=2, P_{R E}=1$ and $P_{L}=1$. Hence

$$
\widetilde{H}=\left[H(0)^{T} H(0)+H(1)^{T} H(1)\right] \text { is known as }
$$

combined matrix which is in the form of $2 \times 2$

$$
\tilde{H}=\left[\begin{array}{cc}
\sum_{l=0}^{1} q_{11}(l)^{2} & \sum_{l=0}^{1} q_{11}(l) q_{12}(l) \\
\sum_{l=0}^{1} q_{11}(l) q_{12}(l) & \sum_{l=0}^{1} q_{12}(l)^{2}
\end{array}\right]
$$

Here, obtaining the diagonal as well as unitary matrix of Eigen values as given below.

$V=\left[\hat{v}_{1} \hat{v}_{2}\right]$ is unitary matrix, where $\hat{v}_{1}$ and $\hat{v}_{2}$ are eigenvector

$S=\left[\begin{array}{cc}s_{1} & 0 \\ 0 & s_{2}\end{array}\right]$ is diagonal matrix. The combined matrix is denoted in the below equation for $\tilde{H}$

$$
\widetilde{H}=\mathrm{VSV} V^{T}
$$

$$
\tilde{H}=\left[\begin{array}{cc}
s_{1} \hat{v}_{11}^{2}+s_{2} \hat{v}_{12}^{2} & s_{1} \hat{v}_{11}^{2} \hat{v}_{21}+s_{2} \hat{v}_{12} \hat{v}_{22} \\
s_{1} \hat{v}_{21} \hat{v}_{11}+s_{2} \hat{v}_{22} \hat{v}_{12} & s_{1} \hat{v}_{21}^{2}+s_{2} \hat{v}_{22}^{2}
\end{array}\right]
$$

By equating the diagonal terms in equation (6) and (8), based on this equations can derive the below relationships

$$
\begin{aligned}
& s_{1} \hat{v}_{11}^{2}=\sum_{l=0}^{1} q_{11}(l)^{2}-s_{2} \hat{v}_{12}^{2} \\
& s_{1} \hat{v}_{21}^{2}=\sum_{l=0}^{1} q_{12}(l)^{2}-s_{2} \hat{v}_{22}^{2}
\end{aligned}
$$

(10)

The eigenvector $\hat{v}_{1}$ contains two elements of $\hat{v}_{11}$ and $\hat{v}_{21}$ and these elements are linked with transmission of first and second antennas. The optimum impact on the eigenvalue is discovered based on the range of each component vector, then remove the corresponding antenna. Where $\hat{u}$ is denoted as antenna index

$$
\hat{u}_{\text {remove }}=\arg \max \left(\hat{v}_{11}^{2}, \hat{v}_{21}^{2}\right)
$$

Above example shows for $2 \times 2$ matrix. Therefore for general case the antenna selection method utilized with $\left(P_{T R} \times P_{T R}\right)$ channel matrix $\tilde{H}\left(=H^{T} H\right)$,

$$
H=\left[H(0)^{T} H(1)^{T} \cdots H(L-1)^{T}\right]^{T}
$$

In antenna selection, a method named as decremental selection strategy is adopted, which begins with set of $\left(P_{L}+1\right)$ transmit antennas. In general case th equation can be written as

$$
\hat{u}_{\text {remove }}=\arg \max \left(\hat{v}_{11}^{2}, \hat{v}_{21}^{2}, \cdots, \hat{v}_{\left(P_{L}+1\right) 1}^{2}\right)
$$

Therefore, set the channel matrix as

$$
H(l)=\left\lfloor q_{(1)}(l), q_{(2)}(l), \cdots, q_{\left(P_{L}+1\right)}(l)\right\rfloor
$$

After, that to determine the least value of combined matrix $\widetilde{H}=\left[(H)^{T} H\right]$ are linked with the eigenvector $\boldsymbol{u}_{1}$, where

$$
H=\left[H(0)^{T} H(1)^{T} \ldots . H(L-1)^{T}\right]^{T}
$$

That transmit antenna, which have highest contribution towards the eigenvalue can removed on the basis of channel $\operatorname{vector} q_{(\hat{u})}(l)$. For the removal of next antenna update the channel $H$ the process is repeated till every transmit antennas $\left(P_{T R}-P_{L}\right)$ are removed.

\section{SOR BASED SIGNAL DETECTION}

For detecting the signal with low complexity, the method of Successive Over Relaxation is utilized. 
This method is used in the signal detection, for eliminating the use of complicated matrix inversion. For uplink, the matrix of MMSE filtering is acting as the symmetric positive definite. Due to this behavior, the equation is generally solved like $\mathrm{Bx}=\mathrm{b}$

Algorithm: Eigen value based transmit antenna selection algorithm

Set

$$
\begin{aligned}
& H(l)=\left\lfloor q_{(1)}(l), q_{(2)}(l), \cdots, q_{\left(P_{L}+1\right)}(L)\right\rfloor, L=0,1, \cdots, L-1 \\
& \text { Compute } \tilde{H}=\left[(H)^{T} H\right] \\
& \text { For } k=1,2, \cdots,\left(P_{T R}-P_{L}\right) \\
& {[U, T]=\operatorname{eig}(\tilde{H})}
\end{aligned}
$$

Find the Eigen vector, $v_{1}$,corresponding to least Eigen value of $\tilde{H}$

$$
\begin{aligned}
& {\left[\hat{v}_{1}, \hat{t}_{1}\right]=\operatorname{sort}\left|v_{1}\right|} \\
& \hat{u}=\hat{r}_{1}^{(1)} \\
& \text { Update } \\
& \left.\underline{H}(l)=\underline{q}_{(1)}(l), \cdots, \underline{q}_{(\hat{u}-1)}(l), \underline{q}_{(\hat{u}+1)}(l), \cdots, \underline{q}_{\left(P_{L}+1\right)}(l)\right] \\
& \text { Define } H=\left[H(0)^{T} H(1)^{T} \ldots . H(L-1)^{T}\right]^{T} \\
& \text { Obtain } \tilde{H}=\left[(H)^{T} H\right] \\
& \text { end } \\
& \tilde{H}_{r}=H
\end{aligned}
$$

in which the B known as the symmetric positive definite matrix. $\mathrm{C}$ is the measurement vector, $\mathrm{x}$ is known as the solution vector, the acquired effectiveness is mentioned by $\mathrm{x}$ based on an iterative way unused any requirement of any inversion of complicated matrix.

The SOR algorithm can be represented as

$$
y^{(j+1)}=\left(L+\frac{1}{\mu} C\right)^{-1}\left[\left(\left(\frac{1}{\mu}-1\right) C-U\right)\right] y^{(j)}+x
$$

Where $\mu$ denotes the relaxation parameter, $\mathrm{j}$ indicates the number of iterations.

A significant role plays by the relaxation parameter in convergence speed, when $\mu=1$, the SOR technique simplifies to the Gauss-Seidel method. Then decompose B as

$$
\mathbf{B}=\mathbf{C}+\mathbf{L}+\mathbf{U}
$$

In this equation, the strictly upper triangular, diagonal component and strictly lower component of the triangle are denoted by $\mathrm{U}, \mathrm{C}$, and $L$. After that apply the method of SOR for estimation of the transmitted signal vector, as expressed below

$$
y^{(j+1)}=\left(L+\frac{1}{\mu} C\right)^{-1}\left[\left[\left(\left(\frac{1}{\mu}-1\right) C-U\right)\right] y^{(j)}+\hat{x}\right]
$$

When $\mathrm{j}=0$ the $\mathrm{y}^{(0)}$ indicates initial solution. SOR fails to convergence if $\mu$ is outside this interval 0 to 2 in large scale MIMO system,

Taking $\quad\left(L+\frac{1}{\mu} C\right)^{-1}\left[\left[\left(\frac{1}{\mu} C-C-U\right)\right]\right]$ and $\left(L+\frac{1}{\mu} C\right)^{-1}$ as $\mathrm{D}$ and then $\left(L+\frac{1}{\mu} C\right)^{-1} \hat{x}$ as $\mathrm{b}$, then the SOR iteration is given as

$$
y^{(j+1)}=D y^{(j)}+b
$$

Based on the representation of Eigen value, expressed as

$$
D_{r}=\left(L+\frac{1}{\mu} C\right)^{-1}\left[\left[\left(\frac{1}{\mu} C-C-U\right)\right]\right] \mathrm{r}=\mathrm{\eta}_{n} r
$$

In this equation $r$ is act as an arbitrary non zero real valued vector, multiplying by $r^{T}$ on both sides

$$
r^{T}\left[\left[\left(\frac{1}{\mu} C-C-U\right)\right]\right] r=\mathrm{\eta}_{n} r^{T}\left(L+\frac{1}{\mu} C\right) r
$$

By taking transpose on both sides;

$$
r^{T}\left[\left[\left(\frac{1}{\mu} C-C-L\right)\right]\right] r=\mathrm{\eta}_{n} r^{T}\left(U+\frac{1}{\mu} C\right) r
$$

Then the equation (21) and (22) are added and the equation can be written as

$$
r^{T}\left[\left[\left(\left(\frac{2}{\mu}-2\right) C-U-L\right)\right]\right] r=\mathrm{\eta}_{n} r^{T}\left(U+L+\frac{2}{\mu} C\right)
$$

Later, substitute the equation (17) into (23) then the equation will be explained as

$$
\left(1-\mathrm{n}_{n}\right)\left(\frac{2}{\mu}-1\right) r^{T} C_{r}=\left(1+\mathrm{n}_{n}\right) r^{T} M_{r}
$$

Hence, this study concluding that SOR iteration is convergent.

\section{A. Symmetric SOR method for Signal detection}

The SSOR method is mostly utilized to conduct the matrix inversion less detection of signal. The symmetric positive definite matrix $W$ is expressed as

$$
W=C+L+L^{T}
$$

Where, lower triangular component denoted as $L$ likewise upper triangular component expressed as $L^{T}$ and $C$ denoted as diagonal component

Then, the first half iteration is calculated and also the iteration known as the method of successive over relaxation.

$$
(C+\mu L) \hat{y}^{(t+1 / 2)}=(1-\mu) C \hat{y}^{(t)}-\mu L^{T} \hat{y}^{(t)}+\mu x
$$

After the first half iteration, the second half iteration done by inverse order

$$
(C+\mu L) \hat{y}^{(t+1)}=(1-\mu) C \hat{y}^{(t+1 / 2)}-\mu L^{T} \hat{y}^{(t+1 / 2)}+\mu x(27)
$$

Based on above equations the iterations are done several times and the obtained vector $\hat{y}$ is multiplied by the matrix. The obtained vector $\hat{y}$ is repeatedly computed for each source signal vector $\mathcal{X}$

\section{B. Proposed improved symmetric SOR methodology for signal detection}

The proposed method is a combination of SOR and SSOR. In this method, four steps are presented

1) Decomposition: In this step the positive definite matrix $W$ of large scale MIMO is decomposed as 


$$
W=C+L+L^{T}
$$

In which, $L^{T} C, L$, are indicated as upper triangular diagonal and lower triangular components of the matrix $W$.

2) First half iteration: The calculation of initial half iteration is conducted which is identical as the SOR method

$$
y^{(t+1 / 2)}=(C+\mu L)^{-1}\left(\mu x+\left((1-\mu) C-\mu L^{T}\right) \hat{y}^{(t)}\right)
$$

Where $t$ indicates that the number of iterations and $\mu$ denotes relaxation parameter.

3) Second half iteration: In this step the second half iteration is calculated with inverse order, which same as the SOR method

$$
y^{(t+1)}=(C+\mu L)^{-1}\left(\mu x+\left((1-\mu) C-\mu L^{T}\right) \hat{y}^{(t+1 / 2)}\right)
$$

4) ISSOR: For the third as well as second step, The ISSOR is weighted then

$$
\hat{y}^{(t+1)}=\theta y^{(t+1 / 2)}+(1-\theta) y^{(t+1)}
$$

A significant role is

The major difference happened in convergence rate as well as convergence by $\theta$ which is known as the weighting factor. When the value of $\theta=1$, the SOR is degenerated by ISSOR method likewise the SSOR degenerated while the value of $\theta=0$ by ISSOR method.

The method of ISSOR will deteriorate into SSOR when $\theta=0$ and into SOR when $\theta=1$. When $0<\mu<2$ the ISSOR based method is feasible in any feasible solution. The major difference can happened in the performance of ISSOR because of the weighting factor as well as relaxation parameter. The relaxation parameter at peak is denoted by

$\mu^{o p t}=\frac{2}{1+\sqrt{2(1-\varphi(A))}}$.

In which, spectral radius of matrix A denoted by $\varphi($.$) and$ the expression is presented by

$$
A=C^{-1} P-I_{K}
$$

The fixed value $\mathrm{N}$ is created for the system of large scale MIMO by the converging of diagonal component $\mathrm{C}$

$$
C^{-1} \approx \frac{1}{N} I_{K}
$$

Where the overall quantity of active users is represented by $\mathrm{K}$,

$\mathrm{K}$ is the quantity of active users \& $\mathrm{N}$ is the quantity of antennas at the base stations. The figure of $\mathrm{K}$ as well as $\mathrm{N}$ is large enough, which $K / N$ will be fixed, the highest then lowest Eigen value of the matrix $\mathrm{H}$ able to be represented as

$$
\eta_{\max }=N\left(1+\sqrt{\frac{K}{N}}\right)^{2}+\gamma
$$

$$
\eta_{\min }=N\left(1-\sqrt{\frac{K}{N}}\right)^{2}+\gamma
$$

$\mu_{1}, \mu_{2}$ are acquired based on the $\eta_{\min }$ and $\eta_{\max }$

Correspondingly

$$
\begin{gathered}
\mu_{1}=\frac{2}{1+\sqrt{2\left(1-d_{1}\right)}} \\
\mu_{2}=\frac{2}{1+\sqrt{2\left(1-d_{2}\right)}}
\end{gathered}
$$

Where $\mathrm{d}_{1}$ and $\mathrm{d}_{2}$ are the spectral radius of the iteration matrix. Then utilize $\bar{\mu}$ relaxation parameter of ISSOR based pre-coding, which equation is represented as

$$
\bar{\mu}=\frac{\mu_{1}+\mu_{2}}{2}
$$

After the continuous experiment this work presented a derivation for $\theta$ which known as the optimal weighting factor

$$
\theta=\left(\frac{K}{N}\right)^{2}
$$

The execution of ISSOR method is generally according to the SSOR method because $\mathrm{N}$ is greater than $\mathrm{K}$ generally in large scale MIMO system.

\section{ENERGY EFFICIENT IN VA-GSM MIMO SYSTEM}

According to the usage of Gaussian codebooks, inter user interference is taking as noise and the exploitation of channel

reciprocity, the average information rate of the $k^{\text {th }} \mathrm{UE}$ is expressed as

$$
I_{r}=\left(1-\frac{K}{T}\right) E\left\{\log _{2}\left(1+\frac{\left|h_{k}^{H} u_{k}\right|^{2}}{\sum_{l=1, l \neq k}^{K}\left|h_{k}^{H} u_{l}\right|^{2}+\sigma^{2}}\right)\right\}
$$

Where $\sigma^{2}$ denoted as noise variance [15]. According to the available Channel state information (CSI) the direction and power of the pre-coding vector $u_{k}$ are calculated. Furthermore, the power utilization of circuit, in addition to the power amplifier at the processing of the signal, is calculated. The below equation expressed to find the consumption of power at the unit of a joule.

$$
P=\sum_{k=1}^{K} \frac{E\left\{\left\|u_{k}\right\|^{2}\right\}}{\lambda}+\sum_{i=0}^{3} C_{i, 0} K^{i}+\sum_{i=0}^{2} C_{i, 1} K^{i} N
$$

The effectiveness of the power amplifier is treated on the basis of the locations of user and channel realizations. $C_{0,0} \geq 0$, represented as consumption of static hardware power. $\lambda$ is the efficiency of power amplifier. 
To consider the energy efficiency (EE) instead of spectral efficiency for the design of communication systems. Hence the system parameters such as transmit power, UE and number of transmitting antennas are optimized for designing energy efficient large scale VA GSM MIMO. Energy efficiency is defined as the ratio between the average information rate and total power consumption.

The average energy efficiency (EE) is given as below,

$$
E E=\frac{\sum_{k=1}^{K} I_{r}}{P}
$$

Then the energy efficiency is maximized based on the assumptions of zero forcing, gives the BS with suitable CSI (channel state information).

\section{A. Optimal system parameters}

Find, which value of the parameters $\mathrm{N}, \mathrm{K}$ and $\rho$ that optimizes the energy efficiency while the other two are fixed. There are $T_{c}$ data samples available in coherence time (T) with the duration of $T$ seconds. The total number of bits transmitted by the K UEs at a unique coherence time. The optimal value of an amount of antennas $\mathrm{N}$ is represented as following

$$
\underset{N \geq K}{\operatorname{maximize}} \frac{K\left(1-\frac{K}{T}\right) \log _{2}(1+\rho(N-K))}{\frac{\rho K B_{\eta}}{\lambda}+\sum_{i=0}^{3} C_{i, 0} K^{i}+\sum_{i=0}^{2} C_{i, 1} K^{i} N}
$$

The optimal transmit power $\rho$ is used for increasing the energy efficiency.

$$
\underset{\rho \geq 0}{\operatorname{maximiz}} \frac{K\left(1-\frac{K}{T}\right) \log _{2}(1+\rho(M-K))}{\frac{\rho K B_{\eta}}{\eta}+\sum_{i=0}^{3} C_{i, 0} K^{i}+\sum_{i=0}^{2} C_{i, 1} K^{i} M}
$$

The optimal number of UEs $K$ is obtained by the transmit power such as $\rho^{\text {tot }}=K \rho$ and number of transmit antennas are fixed as $\alpha=\frac{N}{K}$. Then the energy optimization problem is given as

$$
\operatorname{maximiz}_{K \geq 0} \frac{K\left(1-\frac{K}{T}\right) \log _{2}\left(1+\rho^{\text {tot }}(\alpha-1)\right)}{\frac{\rho K B_{\eta}}{\lambda}+\sum_{i=0}^{3} C_{i, 0} K^{i}+\sum_{i=0}^{2} C_{i, 1} \alpha K^{i} N}
$$

Obtained expressions from the above mentioned other two parameters are fixed. After that the standard alternating optimization are utilized,

1. According to the equation (46) update the number of UEs $K$;

2. Based on the optimal value from equation (44) the

3. On the basis of equation (45) transmit power have to optimize;

4. Repeat these till reaches the convergence.

\section{EXPERIMENTAL RESULTS} equations separately optimizes the $N, K, \rho$ while the number of transmit antennas $N$ have to replace;

The proposed Eigen value based antenna selection and ISSOR based signal detection for large scale VA GSM MIMO system is performed in MATLAB software. For the simulation of this work transmit antennas and receive antennas are 64 and 16 respectively. Then the active antennas are fixed as 4 and number of iterations is 25 . Based on these parameters the output of our proposed work is obtained. Finally our proposed work performance based on bit error rate (BER) is compared with Richardson method, MMSE, SOR and SSOR.

Table 1. Simulation parameters

\begin{tabular}{|c|c|}
\hline Parameters & Values \\
\hline Number of iterations, $\mathrm{t}$ & 25 \\
\hline Coherence time, $\mathrm{T}$ & $32 \mathrm{~ms}$ \\
\hline Noise variance, $\sigma^{2}$ & $10^{-20}$ \\
\hline $\begin{array}{c}\text { Number of antennas, } \\
\mathrm{N}\end{array}$ & 64 \\
\hline $\begin{array}{c}\text { active user equipment, } \\
\mathrm{K}\end{array}$ & $2 \mathrm{dBm}$ \\
\hline $\begin{array}{c}\text { Transmit power, } \rho \\
\text { Number of receive } \\
\text { antennas }\end{array}$ & 16 \\
\hline
\end{tabular}

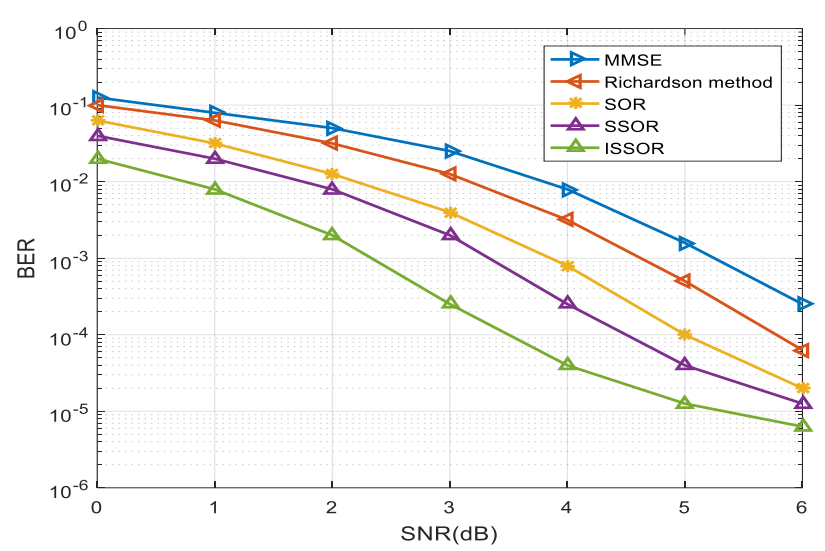

Fig.2 Comparison of BER performance for $N X K=64 X 4$ versus SNR (dB).

Figure 2 shows the BER performance for $N=64$ number of antennas and $K=4$ active users.

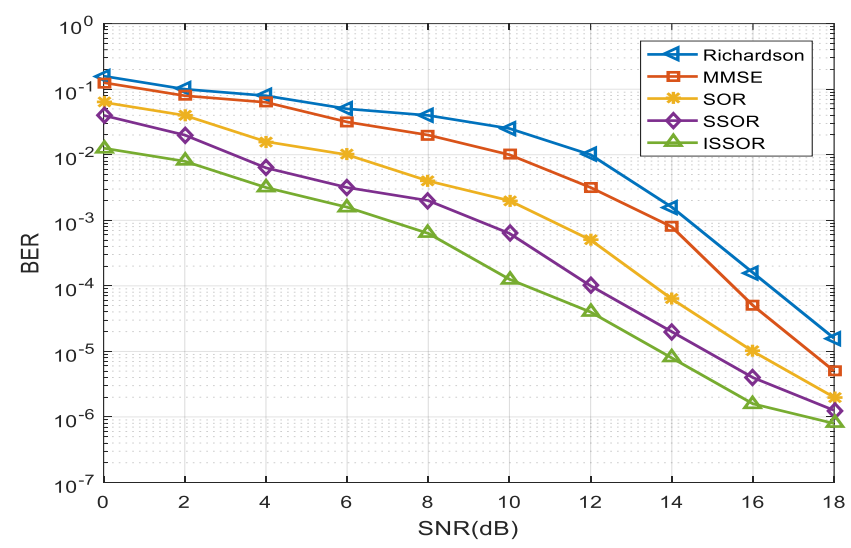

Fig. 3 Comparison of BER performance for $N X K=128 X 4$ versus SNR. 
Figure 3 shows the BER performance of $N=128$ number of antennas and $\mathrm{K}=4$ active users based on our proposed work compared with other existing works such as Richardson method, MMSE and SOR method versus SNR. When the SNR value is low then BER performance of the compared works are high. When the SNR value is increasing the BER rate is decreasing. This graph shows that our proposed ISSOR method achieves good performance when compared to other works.

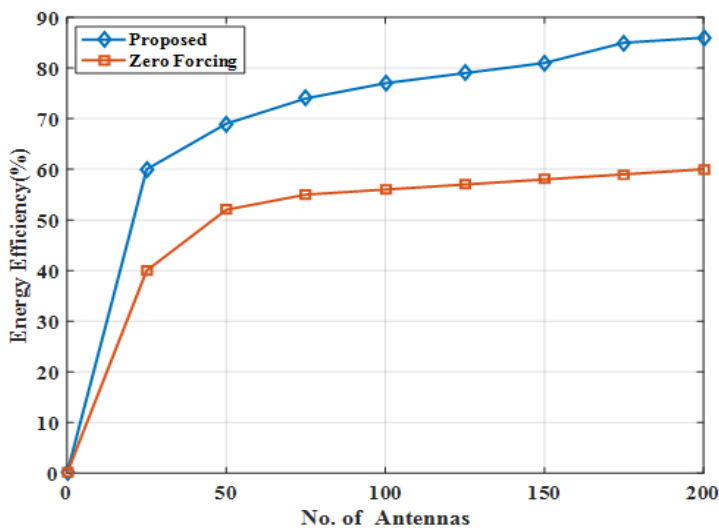

Fig. 4 Comparison of Energy efficiency for ISSOR method and Zero forcing versus No. of antennas

This graph shows that the performance of our proposed work and compared that with zero forcing. When the number of antennas is 50 then our proposed work energy efficiency is $60 \%$ and zero forcing method energy efficiency is $40 \%$. When number of antennas is 100 then our proposed work energy efficiency is $75 \%$ and zero forcing energy efficiency is $55 \%$. The graph shows our proposed work has best performance compared with zero forcing.

\section{CONCLUSION}

Recently the number of antennas used in wireless communication systems are gradually increasing because of that less signal detection and high power consumption is occurred. In this work, proposed and executed a new method for efficient signal detection named as Improved symmetric successive over relaxation (ISSOR) method. Eigen value method is used for antenna selection of our proposed work in large scale MIMO systems. This work executes the detection signal without complex matrix inversion. This work also executes the optimum energy efficiency. The proposed large scale VA GSM MIMO system is implemented in MATLAB platform. After the execution our proposed work is compared with other existing works based on BER. The simulation results shows that our proposed work achieves good performance compared with existing works.

\section{REFERENCES}

1. Zheng, Kan, et al., "Survey of large-scale MIMO systems." IEEE Communications Surveys \& Tutorials vol. 17, no. 3, pp. 1738-1760, (2015).

2. S. Zhao, et al., "A comparative study of low-complexity MMSE signal detection for massive MIMO systems". KSII Transactions on Internet and Information Systems vol. 12, no.4, pp. 1504-1526 (April 2018).

3. Dai, Linglong, et al., "Low-complexity soft-output signal detection based on Gauss-Seidel method for uplink multiuser large-scale MIMO systems." IEEE Transactions on Vehicular Technology vol. 64, no.10 pp. 4839-4845 (2015).
4. Younis, A. Hamid, et al., "Generalised spatial modulation." Signals, Systems and Computers (ASILOMAR), 2010 Conference Record of the Forty Fourth Asilomar Conference on. IEEE, (2010).

5. Wang, Jintao, S. Jia and J. Song, "Generalised spatial modulation system with multiple active transmit antennas and low complexity detection scheme." IEEE Transactions on Wireless Communications vol. 11, no. 4, pp. 1605-1615 (2012).

6. Arévalo, Leonel, et al. "Decoupled signal detection for the uplink of massive MIMO in 5G heterogeneous networks." EURASIP Journal on Wireless Communications and Networking vol. 2017, no. 1, pp. 131 (2017).

7. Gao, Xinyu, et al. "Matrix inversion-less signal detection using SOR method for uplink large-scale MIMO systems." Global Communications Conference (GLOBECOM), IEEE, (2014).

8. Hu, Yuting, et al. "Low-complexity signal detection using CG method for uplink large-scale MIMO systems." Communication Systems (ICCS), 2014 IEEE International Conference on. IEEE, (2014).

9. Z. Zhang, X. Dai, T.T. Liu, "A low-complexity signal detection utilizing AOR iterative method for massive MIMO system." Published in China Communications 2017 DOI:10.1109/cc.2017.8233666

10. Shao, Lin, and Y. Zu, "Approaches of approximating matrix inversion for zero-forcing pre-coding in downlink massive MIMO systems." Wireless Networks pp. 1-6 (2017).

11. Lopes, Hugo, and N. Souto, "Iterative Signal Detection for Large Scale GSM-MIMO Systems." IEEE Transactions on Vehicular Technology (2018).

12. He, Xiaochen, Q. Guo, J. Tong, J. Xi and Y. Yu, "Low-complexity approximate iterative LMMSE detection for large-scale MIMO systems." Digital Signal Processing, Elsevier, vol. 60, pp. 134-139 (2017).

13. Ren, Bin, Y. Wang, S. Sun, Y. Zhang, X. Dai and K. Niu, "Low-complexity MMSE-IRC algorithm for uplink massive MIMO systems." Electronics Letters, IET vol. 53, no. 14, pp. 972-974, (2017)

14. Gao, Zhen, L. Dai, C. Qi, C. Yuen and Z. Wang, "Near optimal signal detector based on structured compressive sensing for massive SM-MIMO." IEEE Transactions on Vehicular Technology vol. 66, no. 2, pp. 1860-1865 (2017)

15. E. Bjornson, L. Sanguinetti, "Designing multi- User MIMO for Energy Efficiency: When is Massive MIMO the Answer?" IEEE WCNC' vol 14 , pp. 242-247.

\section{AUTHORS PROFILE}

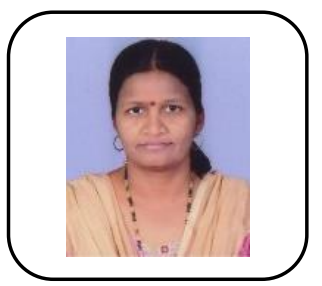

Seema M. Hanchate received the M.E.degree in Control system from the Walchand college of Engineering, Sangli Maharastra, India, in 1999. She is currently pursuing Ph.D.in the Dept. of Electronics and Communication at Usha Mittal Institute of Technology, (UMIT) SNDT Women's University Mumbai, India.

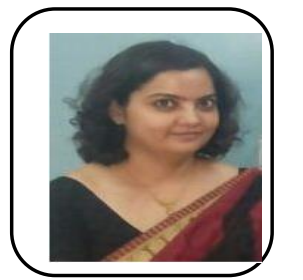

Shikha Nema received her $\mathrm{PhD}$ degree in 2010 and M. Tech degree in Digital Communication from Maulana Azad National Institute of Technology in 2002. She is working as Professor and HOD in electronics and communication department at Usha Mittal Institute of Technology, (UMIT) SNDT Women's University Mumbai. She has published over 40 papers in various International and national journals and conferences. She has guided over 20 M.E and 15 B.E. projects. Currently she is guiding Doctoral students. 